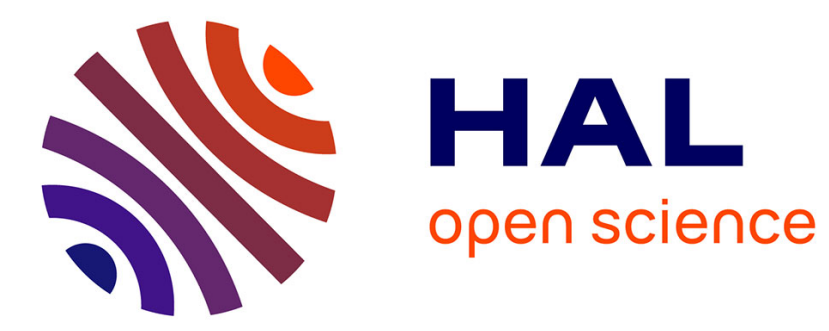

\title{
A Global Constraint for Closed Frequent Pattern Mining
} Nadjib Lazaar, Yahia Lebbah, Samir Loudni, Mehdi Maamar, Valentin Lemière, Christian Bessiere, Patrice Boizumault

\section{To cite this version:}

Nadjib Lazaar, Yahia Lebbah, Samir Loudni, Mehdi Maamar, Valentin Lemière, et al.. A Global Constraint for Closed Frequent Pattern Mining. CP 2016 - 22nd International Conference on Principles and Practice of Constraint Programming, Sep 2016, Toulouse, France. pp.333-349, 10.1007/978-3319-44953-1_22. lirmm-01374719

\section{HAL Id: lirmm-01374719 \\ https://hal-lirmm.ccsd.cnrs.fr/lirmm-01374719}

Submitted on 15 Oct 2018

HAL is a multi-disciplinary open access archive for the deposit and dissemination of scientific research documents, whether they are published or not. The documents may come from teaching and research institutions in France or abroad, or from public or private research centers.
L'archive ouverte pluridisciplinaire HAL, est destinée au dépôt et à la diffusion de documents scientifiques de niveau recherche, publiés ou non, émanant des établissements d'enseignement et de recherche français ou étrangers, des laboratoires publics ou privés. 


\title{
A Global Constraint for Closed Frequent Pattern Mining
}

\author{
N. Lazaar ${ }^{1}$, Y. Lebbah ${ }^{2}$, S. Loudni ${ }^{3}$, M. Maamar ${ }^{1,2}$, \\ V. Lemière ${ }^{3}$, C. Bessiere ${ }^{1}$, P. Boizumault ${ }^{3}$ \\ ${ }^{1}$ LIRMM, University of Montpellier, France \\ ${ }^{2}$ LITIO, University of Oran 1 Ahmed Ben Bella, Algeria \\ ${ }^{3}$ GREYC, Normandie University, Caen, France
}

\begin{abstract}
Discovering the set of closed frequent patterns is one of the fundamental problems in Data Mining. Recent Constraint Programming (CP) approaches for declarative itemset mining have proven their usefulness and flexibility. But the wide use of reified constraints in current $\mathrm{CP}$ approaches leads to difficulties in coping with high dimensional datasets. In this paper, we proposes the ClosedPATtern global constraint to capture the closed frequent pattern mining problem without requiring reified constraints or extra variables. We present an algorithm to enforce domain consistency on CLOSEDPATTERN in polynomial time. The computational properties of this algorithm are analyzed and its practical effectiveness is experimentally evaluated.
\end{abstract}

\section{Introduction}

Frequent Pattern Mining is a well-known and perhaps the most popular research field of data mining. Originally introduced by Agrawal et al. [1], it plays a key role in many data mining applications. These applications include the discovery of frequent itemsets and association rules [1], correlations [2] and many other data mining tasks.

In practice, the number of frequent patterns produced is often huge and can easily exceed the size of the input dataset. Most frequent patterns are redundant and can be derived from other found patterns. Hence, closed frequent patterns have been introduced. They provide a concise and condensed representation that avoids redundancy. Discovering the set of closed frequent patterns is one of the fundamental problems in Data Mining. Several specialized approaches have been proposed to discover closed frequent patterns (e.g., A-ClOSE algorithm [12], CHARM [17], CLOSET [13], LCM [14]).

Over the last decade, the use of the Constraint Programming paradigm (CP) to model and to solve Data Mining problems has received considerable attention $[3,5,9]$. The declarative aspect represents the key success of the proposed $\mathrm{CP}$ approaches. Doing so, one can add/remove any user-constraint without the need of developing specialized solving methods.

Related to the Closed Frequent Pattern Mining problem (CFPM), Guns et. al., propose to express the different constraints that we can have in Pattern Mining as a CP model [5]. The model is expressed on Boolean variables representing 
items and transactions, with a set of reified sum constraints. The reified model has become a de facto standard for many DM tasks. Indeed, the reified model had been adopted for the $k$-pattern sets [5]. The drawback is the wide use of reified constraints in the $\mathrm{CP}$ model, which makes the scalability of the approach questionable.

In the line of the work of Kemmar et al. [8], we propose in this paper the ClosedPattern global constraint. ClosedPattern does not require reified constraints and extra variables to encode and propagate the CFPM problem. ClosedPattern captures the particular semantics of the CFPM problem and domain consistency can be achieved on it using a polynomial algorithm. Experiments on several known large datasets show that our approach outperforms the reified model used in CP4IM [3] and is more scalable, which is a major issue for $\mathrm{CP}$ approaches. This result can be explained by the fact that CLOSEDPATTERN insures domain consistency.

The paper is organized as follows. Section 2 recalls preliminaries. Section 3 provides the context and the motivations for the CLOSEDPATTERN global constraint. Section 4 presents the global constraint ClosedPATTERn. Section 5 illustrates the power of the prunning algorithm compared with the reified model. Section 6 reports experiments. Finally, we conclude and draw some perspectives.

\section{Background}

In this section, we introduce some useful notions used in closed frequent pattern mining and constraint programming.

\subsection{Closed frequent pattern mining}

Let $\mathcal{I}=\{1, \ldots, n\}$ be a set of $n$ item indices ${ }^{1}$ and $\mathcal{T}=\{1, \ldots, m\}$ a set of $m$ transaction indices. A pattern $P$ (i.e., itemset) is a subset of $\mathcal{I}$. The language of patterns corresponds to $\mathcal{L}_{\mathcal{I}}=2^{\mathcal{I}}$. A transaction database is a set $\mathcal{D} \subseteq \mathcal{I} \times \mathcal{T}$. The set of items corresponding to a transaction identified by $t$ is denoted by $\mathcal{D}[t]=\{i \mid(i, t) \in \mathcal{D}\}$. A transaction $t$ is an occurrence of some pattern $P$ iff the set $\mathcal{D}[t]$ contains $P$ (i.e., $P \subseteq \mathcal{D}[t]$ ).

The cover of $P$, denoted by $\mathcal{T}_{\mathcal{D}}(P)$, is the set of transactions containing $P$, that is, $\mathcal{T}_{\mathcal{D}}(P)=\{t \in \mathcal{T} \mid P \subseteq \mathcal{D}[t]\}$. Given $S \subseteq \mathcal{T}$ a subset of transactions, $\mathcal{I}_{\mathcal{D}}(S)$ is the set of common items of $S$, that is, $\mathcal{I}_{\mathcal{D}}(S)=\bigcap_{t \in S} \mathcal{D}[t]$. The (absolute) frequency of a pattern $P$ is the size of its cover (i.e., freq $(P)=\left|\mathcal{T}_{\mathcal{D}}(P)\right|$ ). Let $\theta \in \mathbb{N}^{+}$be some given constant called a minimum support. A pattern $P$ is frequent if $\operatorname{freq}_{\mathcal{D}}(P) \geq \theta$.

Example 1. Consider the transaction database in Table 1. We have $\mathcal{T}_{\mathcal{D}}(C)=$ $\left\{t_{1}, t_{3}\right\}$, freq $_{\mathcal{D}}(C)=2$ and $\mathcal{I}_{\mathcal{D}}\left(\left\{t_{1}, t_{3}\right\}\right)=C H$.

\footnotetext{
${ }^{1}$ For the sake of readability, our examples refer to items by their names instead of their indices.
} 
Table 1: A transaction database $\mathcal{D}$ (a) and its binary matrix (b).

(a)

\begin{tabular}{ccccccccc}
\hline Trans. & & \multicolumn{5}{c}{ Items } & & \\
\hline$t_{1}$ & & $\mathrm{~B}$ & $\mathrm{C}$ & & & & $\mathrm{G}$ & $\mathrm{H}$ \\
$t_{2}$ & $\mathrm{~A}$ & & & $\mathrm{D}$ & & & & \\
$t_{3}$ & $\mathrm{~A}$ & & $\mathrm{C}$ & $\mathrm{D}$ & & & & $\mathrm{H}$ \\
$t_{4}$ & $\mathrm{~A}$ & & & & $\mathrm{E}$ & $\mathrm{F}$ & & \\
$t_{5}$ & & $\mathrm{~B}$ & & & $\mathrm{E}$ & $\mathrm{F}$ & $\mathrm{G}$ & \\
\hline
\end{tabular}

(b)

\begin{tabular}{ccccccccc}
\hline Trans. & $A$ & $B$ & $C$ & $D$ & $E$ & $F$ & $G$ & $H$ \\
\hline$t_{1}$ & 0 & 1 & 1 & 0 & 0 & 0 & 1 & 1 \\
$t_{2}$ & 1 & 0 & 0 & 1 & 0 & 0 & 0 & 0 \\
$t_{3}$ & 1 & 0 & 1 & 1 & 0 & 0 & 0 & 1 \\
$t_{4}$ & 1 & 0 & 0 & 0 & 1 & 1 & 0 & 0 \\
$t_{5}$ & 0 & 1 & 0 & 0 & 1 & 1 & 1 & 0 \\
\hline
\end{tabular}

The closure of a pattern $P$ in $\mathcal{D}$, denoted by $\operatorname{Clos}(P)$, is the set of common items of its cover $\mathcal{T}_{\mathcal{D}}(P)$, that is, $\operatorname{Clos}(P)=\mathcal{I}_{\mathcal{D}}\left(\mathcal{T}_{\mathcal{D}}(P)\right)$. A pattern is closed if and only if $\operatorname{Clos}(P)=P$.

Definition 1 (Closed Frequent Pattern Mining (CFPM)). Given a transaction database $\mathcal{D}$ and a minimum support threshold $\theta$, the closed frequent pattern mining problem is the problem of finding all patterns $P$ such that $\left(\operatorname{freq}_{\mathcal{D}}(P) \geq \theta\right)$ and $(\operatorname{Clos}(P)=P)$.

Example 2. For $\theta=2$, the set of closed frequent patterns in Table 1 is $\emptyset\langle 5\rangle,{ }^{2}$ $A\langle 3\rangle, A D\langle 2\rangle, B G\langle 2\rangle, C H\langle 2\rangle$ and $E F\langle 2\rangle$.

Closed frequent patterns provide a minimal representation of frequent patterns, i.e. we can derive all frequent patterns with their exact frequency value from the closed ones [12]. We now define the important notion of full extension that comes from pattern mining algorithms and that we will use later in this paper.

Definition 2 (Full extension). The non-empty itemset $Q$ is called a full extension of $P$ iff $\mathcal{T}_{\mathcal{D}}(P)=\mathcal{T}_{\mathcal{D}}(P \cup Q)$.

Definition 2 is at the key of the item merging property [15] stated as follows: If some pattern $Q$ is a full extension of some pattern $P$, and none of the proper supersets of $Q$ is a full extension of $P$, then $P \cup Q$ forms a closed pattern. In other words, a closed pattern can be defined as a pattern that does not possess a full extension.

Search Space Issues. In pattern mining, the search space contains $2^{\mathcal{I}}$ candidates. Given a large number of items $\mathcal{I}$, a naive search that consists of enumerating and testing the frequency of pattern candidates in a dataset is infeasible. The main property exploited by most algorithms to reduce the search space is that frequency is monotone decreasing with respect to extension of a set.

Property 1 (Anti-monotonicity of the frequency). Given a transaction database $\mathcal{D}$ over $\mathcal{I}$, and two patterns $X, Y \subseteq \mathcal{I}$. Then, $X \subseteq Y \Rightarrow \operatorname{freq}_{\mathcal{D}}(Y) \leq \operatorname{freq}_{\mathcal{D}}(X)$.

Hence, any subset (resp. superset) of a frequent (resp. infrequent) pattern is also a frequent (resp. infrequent) pattern.

\footnotetext{
${ }^{2}$ Value between $\langle$.$\rangle indicates the frequency of a pattern.$
} 


\subsection{CFPM under constraints}

Constraint-based pattern mining aims at extracting all patterns $P$ of $\mathcal{L}_{\mathcal{I}}$ satisfying a query $q(P)$ (conjunction of constraints), which usually defines what we call a theory [10]: $\operatorname{Th}(q)=\left\{P \in \mathcal{L}_{\mathcal{I}} \mid q(P)\right.$ is true $\}$. A common example is the frequency measure leading to the frequent pattern constraint. It is also possible to have other kind of (user-)constraints. For instance, constraints on the size of the returned patterns, $\min \operatorname{Size}\left(P, \ell_{\min }\right)$ constraint holds if and only if the number of items of $P$ is greater than or equal to $\ell_{\text {min }}$. Constraints on the presence of an item in a pattern $\operatorname{item}(P, i)$ state that an item $i$ must be in a pattern $P$.

\subsection{CSP and global constraints}

A constraint network is defined by a set of variables $X=\left\{x_{1}, \ldots, x_{n}\right\}$, each variable $x_{i} \in X$ having an associated finite domain $\operatorname{dom}\left(x_{i}\right)$ of possible values, and a set of constraints $\mathcal{C}$ on $X$. A constraint $c \in \mathcal{C}$ is a relation that specifies the allowed combinations of values for its variables $X(c)$. An assignment $\sigma$ is a mapping from variables in $X$ to values in their domains. The Constraint Satisfaction Problem (CSP) consists in finding an assignment satisfying all constraints.

Domain Consistency (DC). Constraint solvers typically use backtracking search to explore the search space of partial assignments. At each assignment, filtering algorithms prune the search space by enforcing local consistency properties like domain consistency. A constraint $c$ on $X(c)$ is domain consistent, if and only if, for every $x_{i} \in X(c)$ and every $d_{i} \in \operatorname{dom}\left(x_{i}\right)$, there is an assignment $\sigma$ satisfying $c$ such that $x_{i}=d_{i}$.

Global constraints are constraints capturing a relation between a non-fixed number of variables. These constraints provide the solver with a better view of the structure of the problem. Examples of global constraints are AllDifferent, Regular and Among (see [7]). Except the case when for a given global constraint a Berge-acyclic decomposition exists, global constraints cannot be efficiently propagated by generic local consistency algorithms, which are exponential in the number of the variables of the constraint. Dedicated filtering algorithms are constructed to achieve polynomial time complexity in the size of the input, i.e., the domains and extra parameters. The aim of this paper is to propose a filtering algorithm for the frequent closed pattern constraint.

\section{Context and Motivations}

This section provides a critical review of ad-hoc specialized methods and CP approaches for CFPM, and motivates the proposition of a global constraint.

Specialized methods for CFPM. CLOSE [12] was the first algorithm proposed to extract closed frequent patterns (CFPs). It uses an apriori-like bottom-up method. Later, Zaki and Hsiao [17] proposed a depth-first algorithm based on a vertical database format e.g. CHARM. In [13], Pei et al. extended the FP-growth method to a method called CLOSET for mining CFPs. Lastly, Uno et al. [14] have 
proposed LCM, one of the fastest frequent itemset mining algorithm. It uses a hybrid representation based on vertical and horizontal representations. The milestone of LCM is a technique called prefix preserving closure extension (PPCE), which allows to generate a new frequent closed pattern from a previously obtained closed pattern. Let us explain the PPCE principle. Consider the closed pattern $P$. Let $P(i)=P \cap\{1, \ldots, i\}$ be the subset of $P$ consisting of items no greater than $i$. The core index of $P$, denoted by $\operatorname{core}(P)$, is the minimum index $i$ such that $\mathcal{T}_{\mathcal{D}}(P(i))=\mathcal{T}_{\mathcal{D}}(P)$. A pattern $Q$ is PPCE of $P$ if $Q=\operatorname{Clos}(P \cup\{i\})$ and $P(i-1)=Q(i-1)$ for an item $i \notin P$ and $i>c o r e(P)$. The completeness of PPCE is guaranteed by the following property: If $Q$ is a nonempty closed itemset, then there is only one closed itemset $P$ such that $Q$ is a PPCE of $P$. The mining process using LCM is a depth first search where at each node we have a closed pattern P. LCM uses the PPCE technique as a branching strategy to jump from a closed pattern to other closed patterns by adding new items. With its specialized depth first search, LCM succeeds to enumerate very quickly the closed patterns. However, if the user considers other (user-)constraints on patterns, the search procedure should be revised. In fact, all these specialized proposals (e.g., Closet, Charm, LCM, etc.), though efficient, are ad-hoc methods suffering from the lack of genericity, since adding new constraints requires new implementations.

Reified constraint model for itemset mining. De Raedt et al. have proposed in [3] a CP model for itemset mining. They show how some constraints (e.g., frequency, maximality, closedness) can be modeled as CSP [11,6]. This modeling uses two sets of Boolean variables $P$ and $T$ : (1) Decision variables: item variables $P_{1}, P_{2}, \ldots, P_{n}$, where $P_{i}=1$ if and only if item $i$ is in the searched pattern; (2) Auxiliary variables: transaction variables $T_{1}, T_{2}, \ldots, T_{m}$, where $T_{t}=1$ if and only if the searched pattern is in $\mathcal{D}[t]$.

The relationship between $P$ and $T$, set of channeling constraints, is modeled by reified constraints stating that, for each transaction $t,\left(T_{t}=1\right)$ iff $P$ is a subset of $\mathcal{D}[t]: \forall t \in \mathcal{T}:\left(T_{t}=1\right) \leftrightarrow \sum_{i \in \mathcal{I}} P_{i}(1-\mathcal{D}[t, i])=0$ (arity $\left.n+1\right)$. The min frequency constraint is modeled us: $\forall i \in \mathcal{I}:\left(P_{i}=1\right) \rightarrow \sum_{t \in T} T_{t} \mathcal{D}[t, i] \geq \theta$ (arity $m+1)$. The closedness constraint is expressed with: $\forall i \in \mathcal{I}:\left(P_{i}=1\right) \leftrightarrow$ $\sum_{t \in T} T_{t}(1-\mathcal{D}[t, i])=0$ (arity $\left.m+1\right)$. Such encoding has a major drawback since it requires $(m+n+n)$ reified constraints of arity $(n+1)$ and $(m+1)$ to encode the whole database. This constitutes a strong limitation especially when it comes to handle very large databases.

We propose in the next section the ClosedPattern global constraint to encode both the minimum frequency constraint and the closedness constraint. This global constraint requires neither reified constraints nor auxiliary variables.

\section{ClosedPattern Constraint}

This section presents the CLOSEDPATTERN global constraint for the CFPM problem. 


\subsection{Definition and filtering}

Let $P$ be the unknown pattern we are looking for. The unknown pattern $P$ is encoded with Boolean item variables $P_{1}, \ldots, P_{n}$. In the rest of the paper we will denote by $\sigma$ the partial assignment obtained from the variables $P_{1}, \ldots, P_{n}$ that have a singleton domain. We will also use the following subsets of items:

- present items: $\sigma^{+}=\left\{j \in 1 . . n \mid P_{j}=1\right\}$,

- absent items: $\sigma^{-}=\left\{j \in 1 . . n \mid P_{j}=0\right\}$,

- other items: $\sigma^{*}=\{1 . . n\} \backslash\left(\sigma^{+} \cup \sigma^{-}\right)$.

$\sigma^{*}$ is the set of free items (non instantiated variables). If $\sigma^{*}=\emptyset$ then $\sigma$ is a complete assignment.

The global constraint CLOSEDPATTERN ensures both the minimum frequency property and the closedness property.

Definition 3 (ClosedPattern global constraint). Let $P_{1}, \ldots, P_{n}$ be binary item variables. Let $\mathcal{D}$ be a transaction database and $\theta$ a minimum support. Given a complete assignment $\sigma$ on $P_{1}, \ldots, P_{n}$, CLOSEDPATTERN $_{\mathcal{D}, \theta}(\sigma)$ holds if and only if freq $q_{\mathcal{D}}\left(\sigma^{+}\right) \geq \theta$ and $\sigma^{+}$is closed.

Example 3. Consider the transaction database of Table 1a with $\theta=2$. Let $P=$ $\left\langle P_{1}, \ldots, P_{8}\right\rangle$ with $\operatorname{dom}\left(P_{i}\right)=\{0,1\}$ for $i \in 1$..8. Consider the closed pattern $A D$ encoded by $P=\langle 10010000\rangle$, where $\sigma^{+}=\{A, D\}$ and $\sigma^{-}=\{B, C, E, F, G, H\}$. ClosedPattern $_{\mathcal{D}, 2}(P)$ holds because $\operatorname{fre}_{\mathcal{D}}(\{A, D\}) \geq 2$ and $\{A, D\}$ is closed.

Let $\sigma$ be a partial assignment of variables $P$ and $i$ a free item. We use the vertical representation of the dataset, denoted $\mathcal{V}_{\mathcal{D}}$ where for each item, the transactions containing it are stored: $\forall i \in \mathcal{I}, \mathcal{V}_{\mathcal{D}}(i)=\mathcal{T}_{\mathcal{D}}(\{i\})$. We denote by $\mathcal{V}_{\mathcal{D}}^{\sigma^{+}}(i)$ the cover of item $i$ within the current cover of a pattern $\sigma^{+}$:

$$
\mathcal{V}_{\mathcal{D}}^{\sigma^{+}}(i)=\mathcal{T}_{\mathcal{D}}\left(\sigma^{+} \cup\{i\}\right)=\mathcal{T}_{\mathcal{D}}\left(\sigma^{+}\right) \cap \mathcal{T}_{\mathcal{D}}(\{i\}) .
$$

We need to define extensible assignments.

Definition 4 (Extensible assignment). Given a constraint ClosedPATTERN ${ }_{\mathcal{D}, \theta}$ on $P_{1}, \ldots, P_{n}$, a partial assignment is said to be extensible if and only if it can be extended to a complete assignment of $P_{1}, \ldots, P_{n}$ that satisfies ClOSEDPATtERN ${ }_{\mathcal{D}, \theta}$.

We show when a partial assignment is extensible with respect to ClosedPATTERN constraint.

Proposition 1. Let $\sigma$ be a partial assignment of variables in $P_{1}, \ldots, P_{n} . \sigma$ is an extensible partial assignment if and only if freq $q_{\mathcal{D}}\left(\sigma^{+}\right) \geq \theta$ and $\nexists j \in \sigma^{-}$such that $\{j\}$ is a full extension of $\sigma$.

Proof. According to the anti-monotonicity property of the frequency (cf. Property 1), if the partial assignment $\sigma$ is infrequent (i.e., $\operatorname{freq}_{\mathcal{D}}\left(\sigma^{+}\right)<\theta$ ), it cannot, under any circumstances, be extended to a closed pattern. 
Given now a frequent partial assignment $\sigma$ (i.e., $\operatorname{freq}_{\mathcal{D}}\left(\sigma^{+}\right) \geq \theta$ ), let us take $j \in \sigma^{-}$such that $\{j\}$ is a full extension of $\sigma$. It follows that $\mathcal{T}_{\mathcal{D}}\left(\sigma^{+}\right)=$ $\mathcal{T}_{\mathcal{D}}\left(\sigma^{+} \cup\{j\}\right)=\mathcal{V}_{\mathcal{D}}^{\sigma^{+}}(j)$. Therefore, $\operatorname{Clos}\left(\sigma^{+}\right)=\operatorname{Clos}\left(\sigma^{+} \cup\{j\}\right)$. Since $\sigma^{+}$without $j\left(j\right.$ being in $\sigma^{-}$) cannot be extended to a closed pattern, the result follows. If there is no item $j \in \sigma^{-}$such that $\{j\}$ is a full extension of $\sigma$, then the current assignment $\sigma$ can be definitely extended to a closed itemset by adopting a full extension to form a closed pattern.

We now give the ClosedPATtern filtering rules by showing when a value of a given variable is inconsistent.

Proposition 2 (ClosedPattern filtering rules). Let $\sigma$ be an extensible partial assignment of variables in $P_{1}, \ldots, P_{n}$, and $P_{j}\left(j \in \sigma^{*}\right)$ be a free variable. The following two cases characterize the inconsistency of the values 0 and 1 of $P_{j}$ :

$-0 \notin \operatorname{dom}\left(P_{j}\right)$ iff: $\{j\}$ is a full extension of $\sigma . \quad$ (rule 1)

$-1 \notin \operatorname{dom}\left(P_{j}\right)$ iff: $\begin{cases}\left|\mathcal{V}_{\mathcal{D}}^{\sigma^{+}}(j)\right|<\theta \quad \vee & \text { (rule 2) } \\ \exists k \in \sigma^{-}, \mathcal{V}_{\mathcal{D}}^{\sigma^{+}}(j) \subseteq \mathcal{V}_{\mathcal{D}}^{\sigma^{+}}(k) . & \text { (rule 3) }\end{cases}$

Proof. Let $\sigma$ be an extensible partial assignment and $P_{j}$ be a free variable.

$0 \notin \operatorname{dom}\left(P_{j}\right):(\Rightarrow)$ Let 0 be an inconsistent value. In this case, $P_{j}$ can only take value 1. It means that $\operatorname{Clos}\left(\sigma^{+}\right)=\operatorname{Clos}\left(\sigma^{+} \cup\{j\}\right)$. Thus, $\mathcal{T}_{\mathcal{D}}\left(\sigma^{+}\right)=$ $\mathcal{T}_{\mathcal{D}}\left(\sigma^{+} \cup\{j\}\right)$. By definition 2, $\{j\}$ is a full extension of $\sigma$.

$(\Leftarrow)$ Let $\{j\}$ be a full extension of $\sigma$, which means that $\operatorname{Clos}\left(\sigma^{+}\right)=\operatorname{Clos}\left(\sigma^{+} \cup\right.$ $\{j\})$ (def. 2). The value 0 is inconsistent where $j$ cannot be in $\sigma^{-}$(prop.1).

$1 \notin \operatorname{dom}\left(P_{j}\right):(\Rightarrow)$ Let 1 be an inconsistent value. This can be the case if the frequency of the current pattern $\sigma^{+}$is set up below the threshold $\theta$ by adding the item $j$ (i.e., $\left|\mathcal{V}_{\mathcal{D}}^{\sigma^{+}}(j)\right|<\theta$ ). Or, $\sigma^{+} \cup\{j\}$ cannot be extended to a closed itemset: this is the case when there exists an item $k \in \sigma^{-}$such that at each time the item $j$ belongs to a transaction in the database, $k$ belongs as well $\left(\mathcal{V}_{\mathcal{D}}^{\sigma^{+}}(j) \subseteq \mathcal{V}_{\mathcal{D}}^{\sigma^{+}}(k)\right)$. Conversly, the lack of $k$ (i.e., $\left.k \in \sigma^{-}\right)$implies the lack of $j$ as well. This means that: $\left(P_{k}=0 \Rightarrow P_{j}=0\right)$.

$(\Leftarrow)$ This is a direct consequence of Proposition 1 .

The first rule takes its origin from item merging [15]. The second rule is a basic rule derived from the property of anti-monotonicity of the frequency (Property 1). To the best of our knowledge, the third rule is a new rule taking its originality from the reasoning made on absent items.

Example 4. Following Example 3, consider a partial assignment $\sigma$ such that the variable $P_{1}$ is set to 0 (item $A$ ). That is, $\sigma^{-}=\{A\}$ and $\sigma^{+}=\emptyset$. Value 1 from $\operatorname{dom}\left(P_{4}\right)$ (item $D$ ) is inconsistent because the lack of $A$ implies the lack of $D$ in $\mathcal{D}$ (i.e., $\mathcal{V}_{\mathcal{D}}^{\sigma^{+}}(D) \subseteq \mathcal{V}_{\mathcal{D}}^{\sigma^{+}}(A)$ ). Let now $P_{1}=0, P_{4}=0$, that is, $\sigma^{-}=\{A, D\}$ and $\sigma^{+}=\emptyset$. If the variable $P_{3}$ is set to 1 (item $C$ ), value 1 from $P_{i}, i=\{2,5,6,7\}$ (items $B, E, F, G)$ is inconsistent because $\left|\mathcal{V}_{\mathcal{D}}^{\sigma^{+}}\left(P_{i}\right)\right|<2$, and value 0 from $P_{8}$ (item $H$ ) is also inconsistent because $\{H\}$ is a full extension of $\sigma$. 


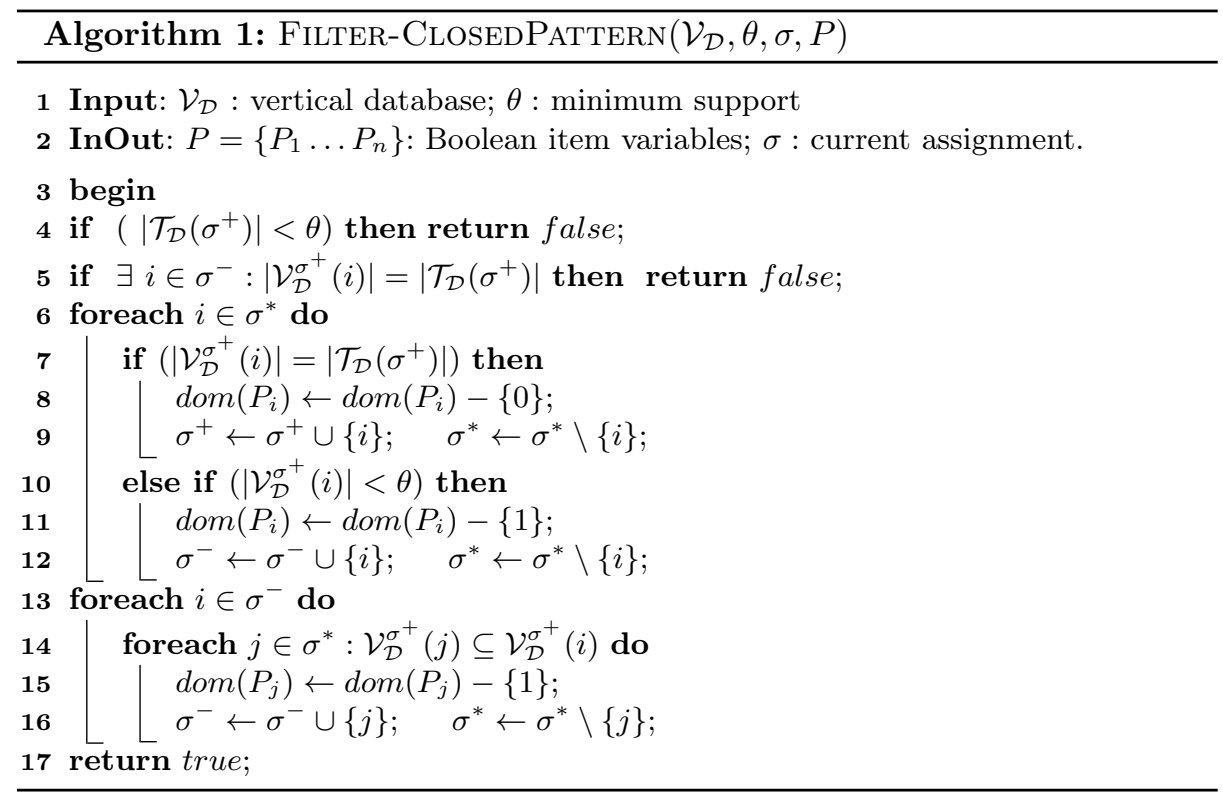

\subsection{ClosedPattern Filtering Algorithm}

In this section, we present the algorithm Filter-ClosedPattern (Algorithm 1) for enforcing domain consistency on the CLOSEDPATTERN constraint. FilteRClosedPattern incrementally maintains the internal data structures $\sigma=<$ $\sigma^{+}, \sigma^{-}, \sigma^{*}>$ and the corresponding cover $\mathcal{T}_{\mathcal{D}}\left(\sigma^{+}\right)$. Using these two structures, one can check if an item is present or not in the vertical dataset $\mathcal{V}_{\mathcal{D}}$.

Algorithm 1 takes as input the vertical dataset $\mathcal{V}_{\mathcal{D}}$, a minimum support threshold $\theta$, the current partial assignment $\sigma$ on $P$ where $\sigma^{*} \neq \emptyset$, and the variables $P$. As output, Algorithm 1 reduces the domains of $P_{i}$ 's and therefore, increases $\sigma^{+}$and/or $\sigma^{-}$, and decreases $\sigma^{*}$.

The algorithm starts by checking if the current partial assignment is extensible or not (Proposition 1). This is performed by checking 1 ) if the size of the current cover is greater than the minimum support (line 4) and 2) if no item of a variable already instantiated to zero is a full extension of $\sigma^{+}$(line 5).

Lines 6-12 are a straightforward application of rules 1 and 2 of Proposition 2. For each non-instantiated variable, 1 ) we check if value 0 is consistent: that item is not a full extension (lines 6-9), and 2) we check if value 1 is consistent: the new cover size by adding that item remains greater than $\theta$ (lines 10-12).

Finally, lines 13-16 implement rule 3 of Proposition 2. We prune value 1 from each free item variable $i \in \sigma^{*}$ such that its cover is a superset of the cover of an absent item $j \in \sigma^{-}\left(\mathcal{V}_{\mathcal{D}}^{\sigma^{+}}(i) \subseteq \mathcal{V}_{\mathcal{D}}^{\sigma^{+}}(j)\right)$.

Theorem 1. Given a transaction database $\mathcal{D}$ of $n$ items and $m$ transactions, and a threshold minsup $\theta$. Algorithm FILTER-CLOSEDPATTERN enforces domain 
consistency on the CLOSEDPATTERN constraint, or proves that it is inconsitent in time $O\left(n^{2} \times m\right)$ with a space complexity of $O(n \times m)$.

Proof. DC: Filter-ClosedPattern implements exactly Proposition 1 and the three rules given in Proposition 2. Thus Filter-ClosedPattern ensures domain consistency (see the description of Algorithm 1).

Time: Let $n=|\mathcal{I}|$ and $m=|\mathcal{T}|$. First, we need to compute $\mathcal{T}_{\mathcal{D}}\left(\sigma^{+}\right)$which requires at most $O(n \times m)$. This is done only once. The cover $\mathcal{V}_{\mathcal{D}}^{\sigma^{+}}(i)$ can be computed by intersecting $\mathcal{T}_{\mathcal{D}}\left(\sigma^{+}\right)$(already computed) and $\mathcal{T}_{\mathcal{D}}(\{i\})$ (given by the vertical representation) within at most $O(m)$. Checking rules 1 and 2 on all free variables can be done in $O(n \times m)$ ( lines 6-12). However, checking rule 3 is cubic at lines 13-16 (i.e., $O(n \times(n \times m)))$, where checking if a cover $\mathcal{V}_{\mathcal{D}}^{\sigma^{+}}(i)$ is a subset of another cover can be done in $O(m)$. Finally, the worst case complexity is $O(n \times(n \times m))$.

Space: The space complexity of FILTER-ClOSEDPATtERn lies in the storage of $\mathcal{V}_{\mathcal{D}}, \sigma$ and the cover $\mathcal{T}$ data structures. The vertical representation $\mathcal{V}_{\mathcal{D}}$ requires at most $n \times m$ space. In the worst case, we have to store $n$ items within $\sigma$ and $m$ transactions within $\mathcal{T}$. That is, the worst case space complexity is $O(n \times m+$ $n+m)=O(n \times m)$.

During the solving process in depth first search, the whole space complexity is $O(n \times(m+n))$ because (1) the depth is at most $n$; (2) $\sigma$ and $\mathcal{T}$ require $O(n \times(m+n)) ;(3)$ the vertical representation is the same data used all along the solving process $O(n \times m) ;(4) O(n \times(m+n))+O(n \times m)=O(n \times(m+n))$.

Proposition 3 (Backtrack-free). Extracting the total number of closed frequent patterns, noted $C$, is backtrack-free with a complexity in $O\left(C \times n^{2} \times m\right)$ using Filter-ClosedPattern to propagate the ClosedPattern constraint.

Proof. Filter-ClosedPattern ensures DC at each node of the search tree. Hence, the closed frequent patterns are guaranteed to be produced in a backtrackfree manner. The explored search tree is a binary full tree where each node is either a leaf (a solution) or possesses exactly two child nodes. The number of nodes is thus in $O(2 \times C)$. Knowing that ensuring DC is in $O\left(n^{2} \times m\right)$, extracting the total number of closed frequent patterns is in $O\left(C \times n^{2} \times m\right)$.

\subsection{Data structures}

To represent the transactional dataset and the cover of items, we adopted the vertical representation format [16]. Our implementation is in or-tools solver ${ }^{3}$. Static structures: for each item $i \in \mathcal{I}, \mathcal{T}_{\mathcal{D}}(i)=\{t \in \mathcal{T} \mid i \in t\}$ is stored as a bitset of size $m$. If $t \in \mathcal{T}_{\mathcal{D}}(i)$, then the associated bit is set to 1 (0 otherwise).

Dynamic structure: a vector $M e m o$ of bitsets is used to store the cover of each partial solution. Memo is a vector of size $n+1$, since at the beginning of the search, the partial assignment is empty. Let $\sigma$ be a partial assignment. Each time a (new) variable $P_{i}$ is instantiated, the cover of the new partial assignment

\footnotetext{
${ }^{3}$ https://developers.google.com/optimization/
} 


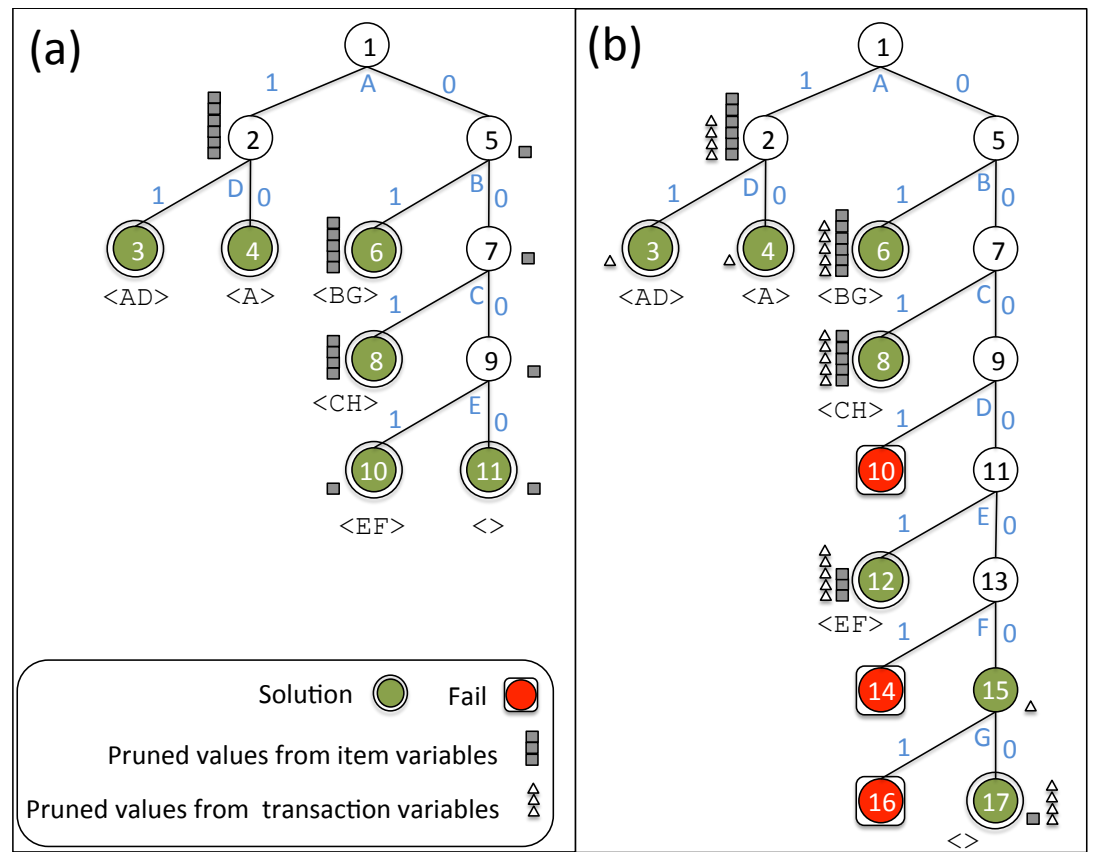

Fig. 1: (a) ClosedPattern and (b) Reified Constraint Model (RCM)

$\sigma \cup\{i\}$ is stored in Memo. If $P_{i}=0$, the cover remains the same: $\mathcal{T}_{\mathcal{D}}\left(\sigma^{+} \cup\{i\}\right)=$ $\mathcal{T}_{\mathcal{D}}\left(\sigma^{+}\right)$. If $P_{i}=1$, the cover of the new partial solution $\sigma \cup\{i\}$ is computed by a bitwise-AND between $\mathcal{T}_{\mathcal{D}}\left(\sigma^{+}\right)$and $\mathcal{T}_{\mathcal{D}}(\{i\})$, and stored in Memo.

Backtracking. First, all $P_{i}$, as well as their domains $\operatorname{dom}\left(P_{i}\right)$, are fully maintained by the or-tools backtracking. Then, a single value (the current index of the vector Memo) is asked to be managed by the or-tools backtracking. Each time a partial solution is extended (from $\sigma$ to $\sigma \cup\{i\}$ ), the current index of Memo is memorised. When a backtrack occurs (from $\sigma \cup\{i\}$ to $\sigma$ ), this value is restored by or-tools giving access to the cover of the (restored) partial solution. Rule 3. The inclusion between two covers $\mathcal{V}_{\mathcal{D}}^{\sigma^{+}}(i) \subseteq \mathcal{V}_{\mathcal{D}}^{\sigma^{+}}(j)$ is rewritten as $\mathcal{V}_{\mathcal{D}}^{\sigma^{+}}(i) \cap \mathcal{V}_{\mathcal{D}}^{\sigma^{+}}(j)=\mathcal{V}_{\mathcal{D}}^{\sigma^{+}}(i)$, and the intersection is performed by a bitwise-AND.

\section{Running example}

In this section, we illustrate the propagation of our CLOSEDPATTERN constraint and the difference that exists comparing to the use of a simple Reified Constraint Model (denoted by RCM, and detailed in Section 3). For that, let us take the transactional dataset given in Table 1: Five transactions $t_{1}$ to $t_{5}$ and eight items from $A$ to $H$.

Figure 1 shows the tree search explored using ClosedPattern (part(a)) and the tree search explored using a reified model $(\operatorname{part}(\mathbf{b}))$ to extract closed 
frequent patterns at minimum support $\theta=2$. Here, both approaches use the same branching heuristics, namely Lex on variables and Max_val on values. First of all, it is worth noticing that the search space that can be explored using ClosedPattern is defined only on decision variables (item variables), whereas the reified model adds a further dimension with auxiliary variables (transaction variables).

At the root node (node 1), no pruning is done since all items are frequent and no item is a full extension of the empty pattern (see Table 1). Thereafter, ClosedPattern and RCM are acting in the same manner on the branch $A=$ 1 . With $A=1, B, C, E, F, G, H$ become infrequent. That is, the 1 values are pruned (rule 2). With RCM on node 2, the pruning on the five decision variables (the item variables) induce a pruning on four auxiliary variables (transaction variables). On the branch $A=1$, two solutions are found: $\langle A D\rangle$ and $\langle A\rangle$.

Branching on $A=0$ (node 5), the value 1 of $D$ is pruned with rule 3 of ClosedPattern. From Table 1, we have $D \Rightarrow A$, and a branching on $A=0$ reduces $D$ to 0 . Here, we can say that the $\mathrm{DC}$ is maintained on node 5 using ClosedPattern, which is not the case using RCM. The same observation can be made on nodes 7,9 and 11 .

Let us take the node 6 , here the branching on $A=0$ and thereafter on $B=1$ will make $C, D, E, F, H$ infrequent (rule 2). Moreover, (rule 1) can be applied since $G$ is a full extension of $B$ (i.e., we cannot have a frequent closed pattern including $B$ without $G$ ). That is, the value 0 is pruned from the domain of $G$, which allows us to reach the solution $\langle B G\rangle$. The same observation can be made on node 8.

To sum up, Filter-ClosedPattern maintains DC at each node and thus, enumerates the solutions backtrack-free (no fails). The same cannot be said with $\mathrm{RCM}$ because the rule 3 is never covered in this example and there are 3 fails.

\section{Experiments}

We made several experiments to compare and evaluate our global constraint with the state of the art methods (CP and specialized methods).

Benchmark datasets. We selected several real and synthetic datasets $[17,4]$ from FIMI repository ${ }^{4}$ with large size. These datasets have varied characteristics representing different application domains. Table 2 reports for each dataset, the number of transactions $|\mathcal{T}|$, the number of items $|\mathcal{I}|$, the average size of transactions $|\widehat{\mathcal{T}}|$, its density $\rho$ (i.e., $|\widehat{\mathcal{T}}| /|\mathcal{I}|$ ) and its size (i.e., $|\mathcal{T}| \times|\mathcal{I}|$ ). We note that the datasets are presented according to their size. They represent various numbers of transactions, numbers of items, densities. We have datasets that are very dense like Chess and Connect (resp. $49 \%$ and 33\%), and others that are very sparse like Retail and BMS-Web-View1 (resp. $0.06 \%$ and $0.5 \%$ ). Note that we have datasets of sizes going from $\approx 10^{5}$ to more than $10^{9}$.

\footnotetext{
${ }^{4}$ http://fimi.ua.ac.be/data/
} 
Table 2: Dataset Characteristics.

\begin{tabular}{|c|c|c|c|c|c|c|}
\hline Dataset & $|\mathcal{T}|$ & $|\mathcal{I}|$ & $|\widehat{\mathcal{T}}|$ & $\rho$ & type of data & size \\
\hline Chess & 3196 & 75 & 37 & $49 \%$ & game steps & 239700 \\
\hline Spl & 3190 & 287 & 60 & $21 \%$ & $\mathrm{~s}$ & 915530 \\
\hline oom & 8124 & 119 & 23 & $19 \%$ & species of mushrooms & 966756 \\
\hline Connect & 67557 & 129 & 43 & $33 \%$ & game steps & $8714 \quad 853$ \\
\hline BMS-Web-View1 & 59602 & 497 & 2.5 & $0.5 \%$ & web click stream & $29622 \quad 194$ \\
\hline T10I4D100K & 100000 & 1000 & 10 & $1 \%$ & synthetic dataset & 100000000 \\
\hline T40I10D100K & 100000 & 1000 & 40 & $4 \%$ & synthetic dataset & 100000000 \\
\hline Pur & 49046 & 7117 & 74 & $1 \%$ & censu & 349060382 \\
\hline Retail & 88162 & 16470 & 10 & $0.06 \%$ & retail market basket data & 1452028140 \\
\hline
\end{tabular}

Experimental protocol. The implementation of our approach was carried out in the or-tools solver. All experiments were conducted on an Intel Xeon E5$2680 @ 2.5 \mathrm{GHz}$ with $128 \mathrm{~Gb}$ of RAM with a timeout of $3600 \mathrm{~s}$. For each dataset, we decreased the (relative) $\theta$ threshold until it is impossible to extract all closed patterns within the allocated time/memory. We have implemented two variants of CLOSEDPATTERN constraint: (i) CLOSEDPATTERN-DC ensuring DC with rules 1,2 and 3 (cubic prunning). (ii) CLOSEDPATTERN-WC ensuring a weaker consistency with only rules 1 and 2 (quadratic prunning). Comparisons are made with: (i) CP4IM, the state-of-the-art on CP approaches, that uses an RCM model. (ii) LCM, the state-of-the-art on specialized methods.

For ClosedPattern and CP4IM, we use the same branching heuristics, namely Lex on variables and Max_val on values. We experimented using the available distributions of LCM-v5.3, ${ }^{5}$ and CP4IM, ${ }^{6}$ with Gecode as the underlying solver of CP4IM. Table 3 gives a comparison between ClosedPatTERN (WC and DC versions), CP4IM and LCM. We report the number of closed patterns \#C of each instance, the number of propagations, the number of nodes, the CPU times in seconds and the number of failures.

ClosedPattern (DC vs WC). Despite the pruning complexity, DC clearly dominates $\mathrm{WC}$ in terms of CPU times (except for BMS1 dataset where both are more or less equivalent). For instance, on the pumsb dataset with $\theta=70 \%$, DC is about 4 times faster than WC. As a second observation, the use of rule 3 can reduce drastically the number of explored nodes and thus, number of propagations. For instance, we note a reduction of $38 \%$ on explored nodes on connect and $98 \%$ on splice 1 compared to WC.

ClosedPattern vs CP4IM. If we compare ClosedPattern with CP4IM, the main observation is that DC outperforms significantly CP4IM at all levels. In terms of CPU times and without counting the Out-of-memory instances, we can observe 23 instances (out of 30) with a speed-up factor between 2 and 15. Factors of 27 to 45 are noted for six instances and for one instance, we have a factor of 182. The weaker version (WC) is also better than CP4IM except two instances in connect and two instances in chess. In terms of number of propagations, we observe a gain factor within a range from 13 to 300. This is because of

\footnotetext{
${ }^{5}$ http://research.nii.ac.jp/ uno/codes.htm

${ }^{6}$ https://dtai.cs.kuleuven.be/CP4IM/
} 
Table 3: ClosedPattern vs CP4IM vs LCM. (oom: Out Of Memory; T0: TimeOut; (1): ClosedPattern-wC; (2): ClosedPattern-dC; (3): CP4IM)

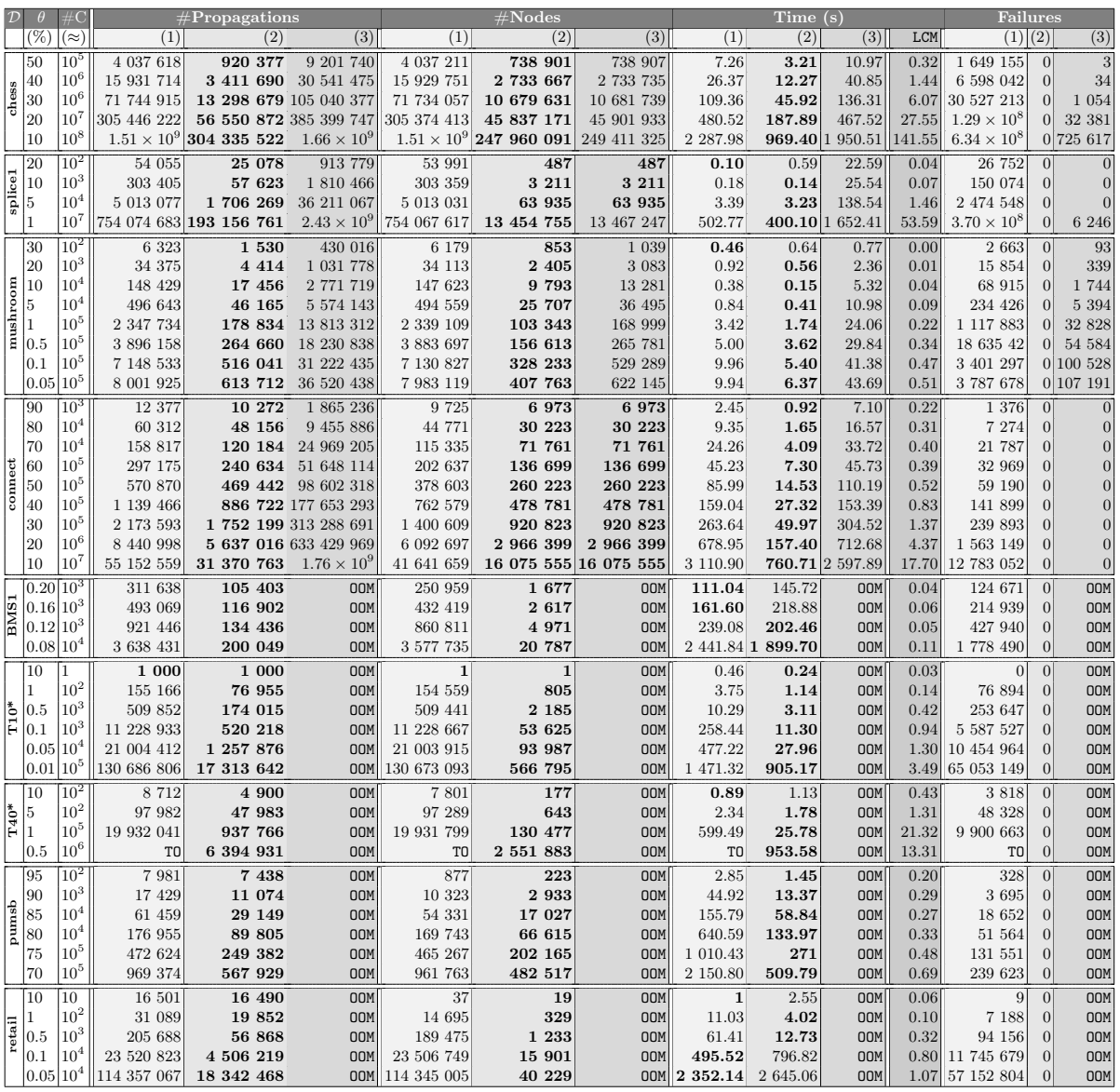

the huge number of propagator calls for reified constraints comparing to one propagator call using CLOSEDPATtern. The number of explored nodes is also reduced, sometimes by half (e.g., mushroom), using ClOSEDPATTERN-DC. This is not a surprise as its number of explored nodes is optimal. Another observation is the experimental validation of Proposition 3: CLOSEDPATTERN-DC extracts the closed patterns in a backtrack-free manner. All solutions are enumerated without any fail (see failures column in Table 3). CP4IM requires an important number of backtracks on most datasets. On connect and three instances of splice1 we can observe that CLOSEDPATTERN-DC and CP4IM explore the same number of nodes. The reified model on such dense datasets and using (Lex \Max_val) heuristics is able to prune all inconsistent values. This result is confirmed by the 
number of failures always equal to zero (backtrack-free). Even if the WC version is faster, CP4IM remains better in terms of pruning (\#nodes). Finally, the major drawback using the reified model for frequent pattern extraction is the memory consumption. We denote 25 Out-of-memory (out of 51 instances). The Out-ofmemory state is due to the huge number of reified constraints. For instance, if we take the T40I10D100K dataset, the CP model produced by CP4IM contains $|\mathcal{T}|+|\mathcal{I}|=101000$ variables, $|\mathcal{T}|=100000$ reified constraints to express the channeling constraints, $2 \times|\mathcal{I}|=2 \times 1000$ reified constraints to express the closure and frequency constraints. This means that the $\mathrm{CP}$ solver has to load in memory a CP model of 102000 reified constraints expressed on 101000 variables, which represents the size given in Table 2. From Table 2, we observe that Gecode is not able to handle CP4IM models on datasets of size greater than $\approx 10^{7}$ (greater than connect dataset).

ClosedPattern vs LCM. In terms of CPU times, LCM remains the leader on basic queries. However, ClosedPATtern is quite competitive as a declarative approach. For instance, if we take chess dataset, LCM is 15 times faster than ClosedPattern-DC on average, where it is more than 120 times faster on average comparing to CP4IM. CLOSEDPATTERN pruning acts only within the current node of the search tree, without imposing any condition on the main search algorithm such as variable or value orderings. This allows to consider new constraints and let the main search algorithm adopt the best heuristics favouring the whole solving. To illustrate our point, we propose to model a particular problem ( $k$-pattern sets) in a declarative manner where LCM could not meet this need.

$k$-patterns instance. A promising road to discover useful patterns is to impose constraints on a set of $k$ related patterns ( $k$-pattern sets) [5,9]. In this setting, the interest of a pattern is evaluated w.r.t. a set of patterns. We propose to model and solve a particular instance, coined dist_kpatterns_lb_ub. Here, we aim at finding $k$ closed patterns $\left\{P^{1}, \ldots, P^{k}\right\}$ s.t:

(i) $\forall i \in[1, k]:$ ClosedPattern $\left(P^{i}\right)$ (Closed Frequent Patterns),

(ii) $\forall i, j \in[1, k]: P^{i} \cap P^{j}=\emptyset$ (all distinct patterns constraints),

(iii) $\forall i \in[1, k]: \mathrm{lb}<\left|P^{i}\right|<\mathrm{ub}$ (min and max size constraints).

Figure 2 shows a comparison between two models, M1 using CLOSEDPATterN for the CFPM part of the problem, M2 using a CP4IM implementation. We selected two dataset instances where CP4IM does not reach the Out-of-memory state and where we have a reasonable number of closed patterns, chess with $\theta=80 \%$ (5084 closed patterns) and connect with $\theta=90 \%$ (3487 closed patterns), and we have varied $k$ with a timeout of $3600 \mathrm{~s}$. After a few preliminary tests, the bounds $\mathrm{lb}$ and $\mathrm{ub}$ on the size of the patterns were set to 2 and 10 respectively. On chess, model M1 is robust and scales well: it is linear on $k$ and never exceeds $6 \mathrm{~min}$ even with $k=12(323.53 \mathrm{~s})$. M2 follows an exponential scale and goes beyond the timeout with only $k=8(7222.41 s)$. The same observation can be made on the connect instance, but in a more pronounced way on the exponential scale followed by M2. With $k=4$, M2 goes beyond the timeout with $5428.05 \mathrm{~s}$ whereas M1 confirms its linear behavior when varying $k$ from 2 to 12 . 
For such problems, a baseline can be the use of specialized methods with postprocessing. One can imagine (i) the use of LCM to extract the total number of closed patterns and (ii) a generate-and-test search trying to find distinct patterns of a given size. Such approach can be very expensive. Here, the postprocessing will generate all the possible $k$ combinations of closed patterns. For instance, we recall that for chess with $\theta=80 \%$ we have 5084 closed patterns. With $k=12$ and using M1, we need less than $6 \mathrm{~min}$, where using the baseline we have to cope with a massive number of combinations. Thus, this last experiment confirms that if LCM is faster on basic queries (e.g., asking for closed frequent with given size), it cannot cope with complex queries. It would need to think and to propose an adhoc solution whereas CP enables a novice DM-user to express his query as constraints.

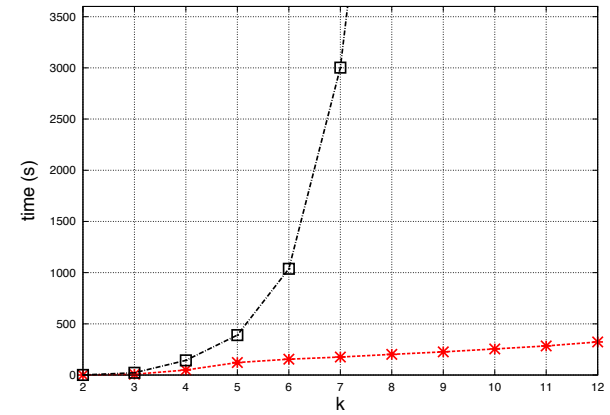

chess $(\theta=80 \%, \mathrm{lb}=2, \mathrm{ub}=10)$

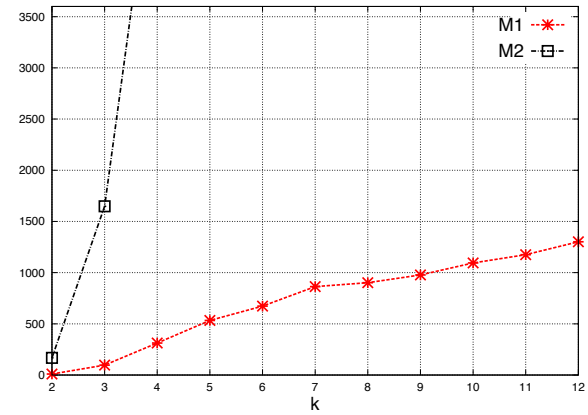

connect $(\theta=90 \%, \mathrm{lb}=2, \mathrm{ub}=10)$

Fig. 2: dist_kpatterns_lb_ub instance using CLOSEDPATTERN and CP4IM.

\section{Conclusion}

In this paper we have introduced a new global constraint for Closed Frequent Pattern Mining. The ClosedPattern constraint captures the particular semantic of the CFPM problem, namely the minimum frequency and closedness of patterns. To propagate efficiently this global constraint, we have first defined three filtering rules that ensure domain consistency. Second, we have defined a filtering algorithm that establishes domain consistency in a cubic time complexity and quadratic space complexity. We have implemented this filtering algorithm into the or-tools solver using a vertical representation of datasets and smart data structures. We have conducted an experimental study on several real and synthetic datasets, showing the efficiency and the scalability of the global constraint compared to a reified constraints approach such as CP4IM. Finally, to show the applicability and the flexibility of CLOSEDPATTERN compared to specialized methods we performed experiments on an instance of $k$-pattern set problem where CLOSEDPATTERN is integrated with a set of constraints. 


\section{References}

1. Agrawal, R., Imielinski, T., Swami, A.N.: Mining association rules between sets of items in large databases. In: Proceedings of the 1993 ACM SIGMOD International Conference on Management of Data, Washington, D.C., May 26-28, 1993. pp. 207216. ACM Press (1993)

2. Brin, S., Motwani, R., Silverstein, C.: Beyond market baskets: Generalizing association rules to correlations. In: SIGMOD. pp. 265-276 (1997)

3. De Raedt, L., Guns, T., Nijssen, S.: Constraint programming for itemset mining. In: Proceedings of the 14th ACM SIGKDD international conference on Knowledge discovery and data mining. pp. 204-212. ACM (2008)

4. Grahne, G., Zhu, J.: Fast algorithms for frequent itemset mining using FP-Trees. IEEE Trans. Knowl. Data Eng. 17(10), 1347-1362 (2005)

5. Guns, T., Nijssen, S., De Raedt, L.: k-pattern set mining under constraints. Knowledge and Data Engineering, IEEE Transactions on 25(2), 402-418 (2013)

6. Guns, T., Nijssen, S., De Raedt, L.: Itemset mining: A constraint programming perspective. Artificial Intelligence 175(12), 1951-1983 (2011)

7. Hoeve, W., Katriel, I.: Global constraints. In: Handbook of Constraint Programming, pp. 169-208. Elsevier Science Inc. (2006)

8. Kemmar, A., Loudni, S., Lebbah, Y., Boizumault, P., Charnois, T.: PREFIXPROJECTION global constraint for sequential pattern mining. In: CP 2015. LNCS, vol. 9255, pp. 226-243. Springer (2015)

9. Khiari, M., Boizumault, P., Crémilleux, B.: Constraint programming for mining n-ary patterns. In: CP 2010. pp. 552-567 (2010)

10. Mannila, H., Toivonen, H.: Levelwise search and borders of theories in knowledge discovery. Data Mining and Knowledge Discovery 1(3), 241-258 (1997)

11. Nijssen, S., Guns, T.: Integrating constraint programming and itemset mining. In: Machine Learning and Knowledge Discovery in Databases, European Conference, ECML PKDD 2010, Barcelona, Spain, September 20-24, 2010, Proceedings, Part II. Lecture Notes in Computer Science, vol. 6322, pp. 467-482. Springer (2010)

12. Pasquier, N., Bastide, Y., Taouil, R., Lakhal, L.: Efficient mining of association rules using closed itemset lattices. Inf. Syst. 24(1), 25-46 (1999)

13. Pei, J., Han, J., Mao, R.: CLOSET: an efficient algorithm for mining frequent closed itemsets. In: SIGMOD Workshop on Data Mining and Knowledge Discovery. pp. 21-30 (2000)

14. Uno, T., Asai, T., Uchida, Y., Arimura, H.: An efficient algorithm for enumerating closed patterns in transaction databases. In: DS 2004. pp. 16-31 (2004)

15. Wang, J., Han, J., Pei, J.: CLOSET+: searching for the best strategies for mining frequent closed itemsets. In: Proceedings of the Ninth ACM SIGKDD International Conference on Knowledge Discovery and Data Mining, 2003. pp. 236-245 (2003)

16. Zaki, M.J., Gouda, K.: Fast vertical mining using diffsets. In: SIGKDD International Conference on Knowledge Discovery and Data Mining. pp. 326-335 (2003)

17. Zaki, M.J., Hsiao, C.: CHARM: an efficient algorithm for closed itemset mining. In: SIAM International Conference on Data Mining. pp. 457-473 (2002) 